\title{
BMJ Open Introducing the non-invasive prenatal test for trisomy 21 in Belgium: a cost-consequences analysis
}

\author{
Mattias Neyt, ${ }^{1}$ Frank Hulstaert, ${ }^{1}$ Wilfried Gyselaers ${ }^{2,3}$
}

To cite: Neyt M, Hulstaert F, Gyselaers W. Introducing the non-invasive prenatal test for trisomy 21 in Belgium: a cost-consequences analysis. BMJ Open 2014;4: e005922. doi:10.1136/ bmjopen-2014-005922

- Prepublication history and additional material is available. To view please visit the journal (http://dx.doi.org/ 10.1136/bmjopen-2014005922).

Received 17 June 2014 Revised 23 September 2014 Accepted 2 October 2014

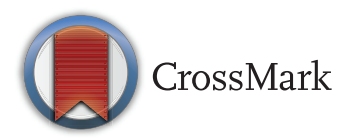

${ }^{1}$ Belgian Health Care Knowledge Centre (KCE), Brussels, Belgium ${ }^{2}$ Department of Obstetrics and Gynaecology, Hospital Oost-Limburg, Genk, Belgium ${ }^{3}$ Hasselt University, Hasselt, Belgium

Correspondence to Dr Mattias Neyt; mattias.neyt@kce.fgov.be

\section{ABSTRACT}

Background: The first- and second-trimester screening for trisomy 21 (T21) are reimbursed for all pregnant women in Belgium. Using a cut-off risk of 1:300 for T21, about $5 \%$ of all pregnant women are referred for definitive prenatal diagnosis using an invasive test, at a sensitivity of (only) $72.5 \%$. The sensitivity and specificity of the non-invasive prenatal test (NIPT) are over $99 \%$ but come at a cost of $€ 460$ (£373) per test. The objective is to estimate the consequences of introducing NIPT for the detection of T21.

Methods: A cost-consequences analysis was performed presenting the impact on benefits, harms and costs. Context-specific real-world information was available to set up a model reflecting the current screening situation in Belgium. This model was used to construct the second and first line NIPT screening scenarios applying information from the literature on NIPT's test accuracy.

Results: Introducing NIPT in the first or second line reduces harm by decreasing the number of procedurerelated miscarriages after invasive testing. In contrast with NIPT in the second line, offering NIPT in the first line additionally will miss fewer cases of T21 due to less false-negative test results. The introduction of NIPT in the second line results in cost savings, which is not true for NIPT at the current price in the first line. If NIPT is offered to all pregnant women, the price should be lowered to about $€ 150$ to keep the screening cost per T21 diagnosis constant.

Conclusions: In Belgium, the introduction and reimbursement of NIPT as a second line triage test significantly reduces procedure-related miscarriages without increasing the short-term screening costs. Offering and reimbursing NIPT in the first line to all pregnant women is preferred in the long term, as it would, in addition, miss fewer cases of T21. However, taking into account the government's limited resources for universal reimbursement, the price of NIPT should first be lowered substantially before this can be realised.

\section{INTRODUCTION}

Prenatal diagnosis of Down syndrome allows for informed decision-making with regard to

\section{Strengths and limitations of this study}

- The major strength of the model is the availability of context-specific real-world information and the ability to reflect the current Belgian screening situation by calibrating the model to the number of women screened, the expected and observed number of children born with Down syndrome and the number of invasive tests performed in Belgium. This calibration assures that the initial screening model reflects the current Belgian screening situation as well as possible.

- The most important limitation of our analysis is that, owing to a lack of reliable data, we were unable to apply a long-term horizon and translate outcomes to incremental cost-effectiveness ratios expressing results in euros per (quality-adjusted) life-year gained. However, by presenting the consequences of screening in a transparent way (which includes the detection of trisomy 21, the number of Down births whether or not after a false-negative screening test, and the number of procedure-related losses), we try to inform policymakers in a transparent way about the possible consequences of introducing NIPT in different settings.

- In order to avoid a 'black box' and to provide other researchers with the possibility to use and adopt the model to their context, details of the full model are included in online supplementary files with a step-by-step explanation for every transition.

pregnancy continuation or termination. Multiple prenatal trisomy 21 (T21, Down syndrome)/aneuploidy screening strategies in the first and second trimesters have been developed. ${ }^{1}$ The most commonly used approach for the first trimester screening in Belgium is the combination of the nuchal translucency (NT) ultrasound measure at week 12 (weeks 11-14), the level of free- $\beta$-human chorionic gonadotrophin hormone and pregnancy associated plasma protein-A, in combination with age and medical history. The T21 screening in Belgium is fully reimbursed for all pregnant 
women and has a high uptake of nearly $80 \%$. However, the overall sensitivity is rather low $(\sim 72.5 \%)$ compared with reports from neighbouring countries. This moderate performance is most likely related to the absence of an obligatory quality assurance system for the NT assessment in Belgium.

The non-invasive prenatal testing (NIPT) is performed on a blood sample of the pregnant woman containing circulating cell free DNA both from the mother and the placenta, which in nearly all cases is representative for the fetal DNA. NIPT has been shown to be highly accurate in the detection of common fetal autosomal trisomies, especially $\mathrm{T}_{21}{ }^{1}$ However, about $4 \%$ of the tests will not provide a result (reduced by half after repeated sampling). The 'no result' NIPT is often caused by a low proportion of fetal DNA, as seen when the sample is obtained before gestational week 12 or in obese women. In dizygotic twin pregnancies, NIPT also remains a challenge. Owing to its high cost, NIPT was originally positioned as a triage test in pregnancies referred for invasive testing (chorionic villus sampling (CVS) or amniocentesis) because of a calculated risk, for example, above 1:300. NIPT for primary screening (at week 12) of pregnant women with an NT under $3.5 \mathrm{~mm}$ is becoming a real possibility in view of the growing number of validation studies in low-risk pregnancies ${ }^{2}$ and especially the prospect of a lower cost per test.

As part of its government-approved work programme, the Belgian Health Care Knowledge Centre (KCE) performed an economic evaluation of introducing NIPT in the prenatal diagnosis of Down syndrome. The research questions were the following: (1) What is the impact of introducing NIPT on the benefits and harms of screening for trisomy 21 in the Belgian context? Benefits can be expressed in terms of detection of trisomy 21 such that informed decision-making is possible. Possible harms in the process include membrane rupture with amniotic fluid leakage or miscarriage after an invasive test, and the risk of missing the detection of Down syndrome because of a false-negative test result. (2) What is the impact on the costs and budget for the health insurance of introducing NIPT? What is the cost for the detection of a case of trisomy 21 after introducing NIPT?

\section{METHODS}

A time-dependent multistage transition probability model was developed in Excel in order to assess the consequences of introducing NIPT. This model allows pregnant women to be followed during the screening process and pregnancy up to birth, taking into account, for example, spontaneous miscarriage rates. In accordance with the Belgian guidelines for economic evaluations, ${ }^{3}$ the analysis includes direct healthcare costs from the perspective of the healthcare payer. Payments out of the public healthcare budget as well as patients' co-payments are included.

A short-term time horizon was applied in which costs and effects before birth were considered. Owing to this short-term horizon, no discount rate was applied. A longterm horizon translating results in extra costs per (quality-adjusted) life-year ((QA)LY) gained was not modelled due to a lack of reliable data and thus the hypothetical character of this scenario. In this cost-consequences analysis, the following outcomes were calculated: total number of live births and number of children born with Down syndrome, cases of T21 diagnosed during pregnancy, children with Down syndrome born after a falsenegative screening result, procedure-related miscarriages (related to T21 detection), short-term screening cost, short-term screening cost per case of T21 diagnosed and incremental cost per extra case of T21 diagnosed.

\section{Population}

The model includes all pregnancies in the Belgian population, except for twin pregnancies. These represent $1.8 \%$ of pregnancies and correspond to about $2.1 \%$ of all T21 cases. $^{4} 5$ Complete and up-to-date data from Flanders, the northern community of Belgium representing $54 \%$ of the children born in Belgium, were extrapolated to the Belgian situation. The model takes into account the different probabilities of a spontaneous loss of the fetus, for T21 and non-T21 pregnancies, adjusted for gestational week (eg, $5 \%$ and $36 \%$ at week 10 for all and T21 pregnancies, respectively (see table 1)). ${ }^{6}{ }^{7} \mathrm{~A}$ total of 122739 births in Belgium in 2012 thus corresponds to 129199 singleton pregnancies at gestational week 10. The observed live birth prevalence of Down syndrome in Belgium, extrapolated from the Flanders registry, was estimated at 98 in 2012, of which 96 were after singleton pregnancies. On the basis of the age distribution of the pregnant women in Flanders and reported age-related prevalence of Down syndrome, ${ }^{8} 219$ T21 singleton live births would be expected without screening, corresponding to 342 pregnancies at week 10 . These numbers of expected and observed births of children with Down syndrome were used to calibrate the model. ${ }^{9}$

\section{Comparators}

The current practice in Belgium for the first or second trimester screening for T21 is modelled and serves as the initial comparator. NIPT is the intervention under consideration and is considered both as a contingent test (ie, as a triage or second line test) and for primary screening (ie, as a first line test). Figure 1 presents the triage scenario in which NIPT is offered only to women at increased risk (>1:300) after the current screening. The risk cut-off is changed in modelled scenario analyses (see the part 'Uncertainty and scenario analyses'). The figures representing the current Belgian screening strategy and NIPT in the first and second line are presented as online supplementary material.

\section{Input variables}

The values and probabilities of all input variables in the models are provided in table 1. Costs for screening, adverse events and pregnancy termination are included 
Table 1 Input variables (volumes and probabilities)

\begin{tabular}{|c|c|c|c|}
\hline Variable & $\begin{array}{l}\text { Mean } \\
(\%)\end{array}$ & Uncertainty & Source \\
\hline Screening uptake & 78.87 & & Belgian data (NIHDI) \\
\hline $\begin{array}{l}\text { Testing uptake (ie, screening+invasive test } \\
\text { without prior screening) }\end{array}$ & 79.74 & Scenario analysis: $90 \%$ & Belgian data (NIHDI) \\
\hline Current screening accuracy & & Scenario analysis+ & Belgian data (AML) \\
\hline Sensitivity & 72.54 & $\beta(103 ; 39)$ & \\
\hline Specificity & 95.03 & $\beta(117144 ; 6121)$ & \\
\hline NIPT & & & Literature $^{10}$ \\
\hline Sensitivity & 99.3 & $\begin{array}{l}95 \% \mathrm{Cl} 98.2 \text { to } 99.8 \%(\beta(6 ; 1.06) ; \\
2.5 \%: 0.982 ; 97.5 \%: 0.998)\end{array}$ & \\
\hline Specificity & 99.84 & $\begin{array}{l}95 \% \text { Cl } 99.69 \text { to } 99.92 \%(\beta(3 ; 1.014) \\
2.5 \%: 0.9969 ; 97.5 \%: 0.9992)\end{array}$ & \\
\hline NIPT test failure rate & & & Expert opinion plus \\
\hline First test (at week 12) & 4 & $\begin{array}{l}\text { Minimum-maximum } 3-7 \%(\beta(2 ; 6) \\
\text { minimum: } 0.03 ; \text { maximum: } 0.07)\end{array}$ & literature $^{10}$ \\
\hline Second test (at week 13) & 2 & $\begin{array}{l}\text { Minimum-maximum: } 1-3 \%(\beta(2 ; 2) \\
\text { minimum: } 0.01 ; \text { maximum: } 0.03)\end{array}$ & \\
\hline $\begin{array}{l}\text { Probability of having an invasive test (after } \\
\text { a positive screening test or NIPT) }\end{array}$ & 87.5 & $\begin{array}{l}\text { Minimum-maximum: } 0.8-0.95 \%(\beta(2 ; \\
\text { 2); minimum: } 0.8 \text {; maximum: } 0.95)\end{array}$ & $\begin{array}{l}\text { Assumption and model } \\
\text { fitting plus literature }\end{array}$ \\
\hline $\begin{array}{l}\text { Number of invasive tests without prior } \\
\text { screening }\end{array}$ & 3212 & $\begin{array}{l}\text { Conditional } \beta \text { distribution (313.9; } 1000 \\
84.1 ; 1814)\end{array}$ & $\begin{array}{l}\text { Belgian NIHDI data and } \\
\text { model fitting; literature }\end{array}$ \\
\hline Invasive testing (CVS or amniocentesis) & & l & Considered as the gold \\
\hline Sensitivity & 100 & & standard \\
\hline Specificity & 100 & & \\
\hline $\begin{array}{l}\text { Procedure-related fetal loss after invasive } \\
\text { test }\end{array}$ & 1 & $\begin{array}{l}\text { Minimum-maximum: } 0.5-2 \%(\beta(2 ; 4) \\
\text { minimum: } 0.005 ; \text { maximum: } 0.02)\end{array}$ & Literature $^{14}$ \\
\hline $\begin{array}{l}\text { Hospitalisation for amniotic fluid leakage } \\
\text { after invasive test }\end{array}$ & 1 & $\begin{array}{l}\text { Minimum-maximum: } 0.5-2 \%(\beta(2 ; 4) \text {; } \\
\text { minimum: } 0.005 ; \text { maximum: } 0.02)\end{array}$ & Literature $^{13}$ \\
\hline Pregnancy termination after T21 diagnosis & 95.45 & $\beta(42 ; 2)$ & $\begin{array}{l}\text { Belgian data and } \\
\text { literature }^{1117}\end{array}$ \\
\hline Spontaneous miscarriage & & & Literature $^{67}$ \\
\hline Miscarriage all $(p)$ & \multicolumn{3}{|c|}{$\begin{array}{l}0.05,0.025,0.015 \text { at weeks } 10,12 \text { and } 14, \\
\text { respectively* }\end{array}$} \\
\hline T21 miscarriage $(p)$ & \multicolumn{3}{|c|}{$\begin{array}{l}0.36,0.3,0.25 \text { at weeks } 10,12 \text { and } 14 \text {, } \\
\text { respectively }\end{array}$} \\
\hline
\end{tabular}

${ }^{*}$ Rounded numbers extracted from a published figure. ${ }^{7}$

AML, Algemeen Medisch Laboratorium bvba; CVS, chorionic villus sampling; NIHDI, National Institute for Health and Disability Insurance; NIPT, non-invasive prenatal test.

and are expressed in $€$ for the year 2013 (table 2). These costs are based on data from our National Institute for Health and Disability Insurance (NIHDI).

On the basis of reimbursement data from NIHDI for the year 2011, excluding the $1.8 \%$ twin pregnancies, 78168 pregnant women participated in the first trimester screening (€80.42 per activity) and another 21451 in the second trimester screening ( $€ 45.03$ per activity). The fee for these activities is exclusive of the ultrasound but includes the counselling which is performed by the healthcare worker offering antenatal screening. NIPT is not a replacement for ultrasound screening and thus no incremental impact on ultrasound screening is included in the model. After adjustments for gestational week, the total screening uptake is estimated at $78.87 \%$. If we also assume 1000 women who immediately undergo invasive testing for T21, the overall uptake of any type of testing for Down syndrome increases to $79.74 \%$. In the reference case, this screening uptake is kept constant.

The sensitivity and specificity of screening at different risk cut-offs are based on the receiver operator characteristics curve data from AML (Algemeen Medisch Laboratorium bvba), a large laboratory covering $40 \%$ of the first and second trimester screening for Down syndrome in Flanders. In the reference case, a risk cut-off level of 1:300 is applied, which results in a sensitivity of $72.54 \%$ (95\% CI $0.649 \%$ to $0.795 \%$ ) and specificity of $95.03 \%$ ( $95 \%$ CI $0.949 \%$ to $0.952 \%$ ). This is varied in the modelled scenario analyses (see the part 'Uncertainty and scenario analyses').

The baseline cost for NIPT (and also for a repeat NIPT if needed) is set at $€ 460$, that is, the current price charged by the University Hospital of Leuven in Belgium. We assume a no first time NIPT result in $4 \%$ 


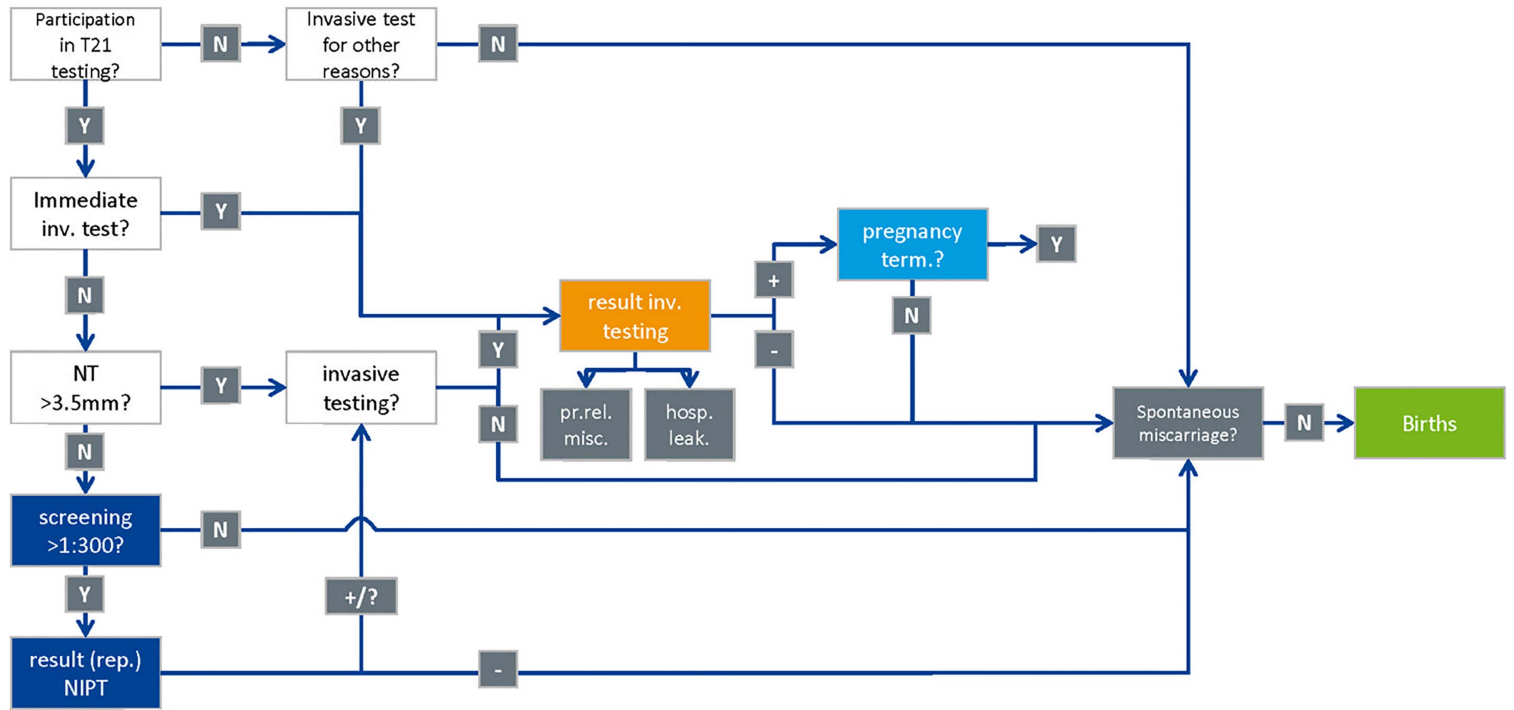

Figure 1 Screening strategy with NIPT as a triage test. NIPT, non-invasive prenatal test; T21, trisomy 21; NT, nuchal translucency; hosp.leak., hospitalisation for leakage; inv., invasive; pr.rel.misc., procedure-related miscarriage; rep., repeat; term.: termination.

(3-7\%) of cases, reduced to $2 \%(1-3 \%)$ after a repeat NIPT. These estimates are in agreement with 11 studies reviewed by Benn et al. ${ }^{10}$ In the primary NIPT model, we assume that $2 \%$ of the women tested will agree to fall back on the current screening and not opt directly for an invasive test. On the basis of an overview of existing evidence, the sensitivity and specificity of NIPT tests with a result are assumed to be $99.3 \%(95 \%$ CI $98.2 \%$ to $99.8 \%$ ) and $99.84 \%$ (95\% CI $99.69 \%$ to $99.92 \%)$, respectively. ${ }^{10}$ No additional cost for NIPT counselling is included since it is assumed that this would happen in a similar way as in the current screening approach and thus does not occur as an incremental cost.

Invasive diagnostic testing is recommended after a positive current screening test or NIPT result in order to confirm the results. The proportion of women undergoing an invasive test after a positive screening was $86.9 \%$ $(95 \%$ CI $83.9 \%$ to $89.5 \%)$ in a large study in Paris. ${ }^{11}$ We use a similar probability of $87.5 \%(80-95 \%)$, which was obtained after model calibration. Having no real-world data at our disposal, this proportion of women undergoing an invasive test is also used in the model after a positive or a 'no result' for NIPT in case of triage, or after a positive NIPT result in case of a first line NIPT. In case of a 'no result' NIPT in the first line, we assume that screening continues with the current approach. The total cost for an invasive procedure and genetic testing for Down syndrome is on average $€ 934$ based on the data of NIHDI.

The total number of invasive tests in Belgium in 2011 is 7586. On the basis of the modelling exercise, 4374 tests are performed following the current screening. On the basis of expert opinion and model calibration, the remaining tests are performed: (1) following an NT>3.5 mm ( $\mathrm{n}=398)$, (2) for other indications (but samples are also tested for T21; n=1814) and (3) in pregnant women who want more certainty without being at increased risk $(n=1000)$. These 1000 women represent

Table 2 Input variables (costs)

\begin{tabular}{|c|c|c|c|}
\hline Variable & Mean & Uncertainty & Source \\
\hline First trimester screening & $€ 80.42$ & I & NIHDI \\
\hline $\begin{array}{l}\text { Second trimester } \\
\text { screening }\end{array}$ & $€ 45.03$ & I & NIHDI \\
\hline NIPT & $€ 460$ & Scenario and threshold analysis & University Hospital Leuven \\
\hline Invasive diagnostic test & $€ 934.21$ & $\begin{array}{l}\text { Minimum-maximum: } € 887.71 ; € 980.71 \\
\text { (uniform) }\end{array}$ & $\begin{array}{l}\text { NIHDI (and expert opinion for the } \\
\text { distribution) }\end{array}$ \\
\hline $\begin{array}{l}\text { Hospitalisation for } \\
\text { leakage }\end{array}$ & $€ 3514.54$ & $\pm 20 \%$ (uniform) & $\begin{array}{l}\text { NIHDI (and expert opinion for the } \\
\text { distribution) }\end{array}$ \\
\hline Pregnancy termination & $€ 914.39$ & $\begin{array}{l}\text { Minimum-maximum: } € 658.24 ; € 1170.54 \\
\text { (uniform) }\end{array}$ & $\begin{array}{l}\text { NIHDI (and expert opinion for the } \\
\text { distribution) }\end{array}$ \\
\hline
\end{tabular}

Exchange rate 22 May 2014: $€ 1=£ 0.81$.

NIHDI, National Institute for Health and Disability Insurance; NIPT, non-invasive prenatal test. 
$0.8 \%$ of all pregnant women, and we assume that no prior screening test is performed or billed. The number of 1000 primary invasive tests is included in all modelled scenarios of the current screening and triage NIPT. However, we assume that these 1000 women will opt for primary NIPT screening once available as NIPT provides more certainty. In Belgium, the samples obtained from invasive procedures are analysed at one of the eight centres for human genetics. The test sensitivity of CVS has been found to be somewhat lower compared with amniocentesis $\left(98.47 \%\right.$ vs $99.32 \%$, respectively). ${ }^{12}$ However, in our model, we assume $100 \%$ accuracy for these last-stage analyses.

Invasive testing carries a risk of membrane rupture with amniotic fluid leakage. ${ }^{13}$ This may lead in about $1 \%$ of procedures to a hospitalisation of about 1 week at a cost of $€ 3515$ and in about $1 \%$ to a procedure-related miscarriage. The latter is based on a Cochrane review which states that "the best estimate of an 'excess' risk after second trimester amniocentesis comes from Tabor 1986. ${ }^{14}$ In a low-risk population with a background pregnancy loss of around 2\%, a mid-trimester amniocentesis will increase this risk by another $1 \% "{ }^{15}$ This miscarriage rate may be more frequent after CVS compared with amniocentesis, and rates are expectedly lower in experienced hands. ${ }^{14}$ It has been reported that $89 \%$ to $97 \%$ of the women who received a positive diagnosis of T21 during the prenatal period had an induced abortion. ${ }^{16}$ Belgian data covering a 10-year period (2003-2012) in a single centre show a diagnosis of T21 after an invasive test during pregnancy in 44 cases. A T21 pregnancy termination was induced in 42 of these 44 cases $(95.45 \%$, $95 \%$ CI $87.7 \%$ to $99.4 \%$ ), which is used in the model. This is in agreement with a proportion of $94.8 \%(95 \%$ CI $92.5 \%$ to $96.5 \%$ ) reported in Paris ${ }^{11}$ and $93.3 \%$ (250 out of 268) in the UK. ${ }^{17}$ Pregnancy termination is associated with a 24-48 h hospitalisation and costs on average $€ 914$.

\section{Uncertainty and scenario analyses}

Both one-way and probabilistic sensitivity analyses were applied. The impact of uncertainty around all the input parameters of the model on the results was modelled probabilistically. The applied distribution depends on the type of variable: ${ }^{18}$ probabilities (eg, NIPT test failure or procedure-related fetal loss) and test characteristics (sensitivity and specificity) were modelled as $\beta$ distributions. This distribution is limited to the $0-1$ scale and reflects the possible outcomes for these variables. For cost variables with less informative data for a stochastic distribution, uniform distributions were applied.

Several one-way scenario analyses are modelled:

- The cut-off risk of 1:300 for T21 is changed to 1:600, 1:1100, 1:1700, 1:2400 and 1:3000.

- A scenario with a $90 \%$ NIPT uptake in the first line (instead of the current uptake with the first and second trimester screening of about $80 \%$ ) is presented without changing any other input variable.
- A threshold analysis is performed, changing the price of NIPT to keep the short-term costs per case of T21 diagnosed at the same level as in the current screening scenario.

- A scenario with improved performance of the current screening (sensitivity of $77.5 \%$ instead of $72.5 \%$ ).

For further details, we refer to the online supplementary file. 1000 Latin Hypercube simulations are performed and correlation coefficients are calculated in a probabilistic sensitivity analysis. The @ Risk add-on tool (Palisade Corporation) is used for probabilistic modelling and sensitivity analyses.

\section{RESULTS}

\section{Reference case}

Table 3 presents the results for the three reference case scenarios. In the current screening situation without NIPT, 170 cases of T21 are diagnosed. In total, 96 children with Down syndrome are born, including 41 after a false-negative screening result. There are 58 iatrogenic miscarriages after T21-related invasive testing. The total short-term costs of screening are almost $€ 15$ million and the short-term average cost per T21 diagnosed is about $€ 87000$.

Introducing NIPT as a triage test (cut-off 1:300) results in one extra case of T21 diagnosis missed after a falsenegative NIPT result. However, there are much less procedure-related miscarriages after T21-related invasive testing (16 vs 58). Both the total short-term costs (minus $€ 1.6$ million) and short-term average cost per case of T21 diagnosed are lower.

Introducing NIPT in the first line results in more cases of T21 being diagnosed ( $\mathrm{n}=215$ vs currently 170 ), very few children with Down syndrome born after a falsenegative screening result ( $\mathrm{n}=2$ vs 41 currently), and a significant decrease in iatrogenic miscarriages related to T21 ( $\mathrm{n}=8$ vs 58 currently). However, at a price of NIPT of $€ 460$, the short-term budget increases to almost $€ 51$ million with a tripled average cost per case of T21 diagnosed at about $€ 236000$. The extra cost per extra case of T21 diagnosed versus NIPT as a triage test is about $€ 840000$.

\section{Uncertainty and scenario analyses}

Figure 2 provides an overview of the most relevant scenarios, including the impact of uncertainty of all input variables. The $\mathrm{x}$-axis and $\mathrm{y}$-axis represent the number of T21 diagnoses and total short-term costs, respectively. We remark that these are not the only outcomes of importance. Other outcomes, such as the number of procedure-related miscarriages, should also be taken into consideration. Further details on all outcomes are mentioned in online supplementary tables.

More patients would receive NIPT in the second line if the risk cut-off after the first and second trimester screening is lowered. As a result, the number of T21 detections would increase and fewer children with Down 


\section{Table 3 Results}

\begin{tabular}{llll}
\hline Test strategy & Current screening & NIPT second line & NIPT first line \\
\hline $\begin{array}{l}\text { (Down) births, diagnosis and miscarriages } \\
\text { Number of births }\end{array}$ & 122543 & 122554 & 122560 \\
Number of Down born & 96 & 97 & 63 \\
Number of Down born (false-negative screening) & 41 & 42 & 2 \\
Number of T21 detected & 170 & 169 & 215 \\
Number of procedure-related miscarriages & 76 & 34 & 26 \\
Number of T21 procedure-related miscarriages & 58 & 16 & 8 \\
Costs for testing during pregnancy & $€ 7252215$ & $€ 7252215$ & $€ 89123$ \\
First and second trimester screening cost & $€ 0$ & $€ 2390929$ & $€ 47969932$ \\
NIPT cost & $€ 7086886$ & $€ 3203417$ & $€ 2435450$ \\
Cost invasive tests & $€ 415728$ & $€ 268375$ & $€ 279539$ \\
Cost hospitalisation for leakage and pregnancy termination & $€ 14754829$ & $€ 13114935$ & $€ 50774045$ \\
Total cost (short term) & $€ 86944$ & $€ 77696$ & $€ 236436$ \\
Short-term cost/T21 detected & $/$ & $€ 2738197^{\star}$ & $€ 839936$ \\
Extra cost per extra T21 detected & & & \\
*This result is located in the third quadrant, that is, fewer cases of T21 diagnosed with a lower cost. The results with their $95 \%$ credibility \\
intervals (Crl) are not presented but are available on request. & & &
\end{tabular}

syndrome would be born after a false-negative screening. The number of procedure-related miscarriages would increase only slightly each time the cut-off risk is lowered. The short-term total screening costs and average cost per T21 detected are lower compared with the current screening situation if NIPT is used as a triage test with a risk cut-off of up to 1:600. However, if the risk cut-off is lowered further, the extra cost per extra T21 detected increases exponentially (figure 2 and table 6 in online supplementary material).

The threshold analysis resulted in a price of about $€ 152$, which would keep the short-term screening cost per T21 diagnosed constant if NIPT is used in the first line. This is illustrated in figure 2. At this price and the current screening uptake of about $80 \%$, we would do much better (more T21 detected, less children born

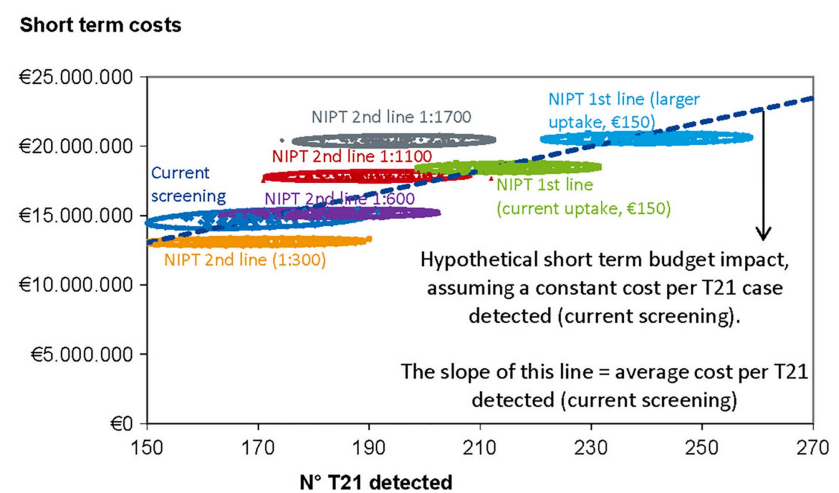

Figure 2 Presentation of most relevant screening scenarios. See the discussion for further explanation on the interpretation of the line presenting the 'average cost per T21 detected (current screening)'. Remark: this figure does not present other outcomes of importance, such as the number of procedure-related miscarriages (NIPT, non-invasive prenatal test; T21, trisomy 21). with Down syndrome after a false-negative screening, and less procedure-related miscarriages). At a constant average cost of about $€ 87000$ per case of T21 diagnosed, this would lead to an increase in the short-term costs, proportional to the increased detection rate (see online supplementary table). The same is shown in figure 2 for a $90 \%$ uptake scenario.

The probabilistic sensitivity analysis showed that the most important stochastic variables in the current screening model and the model with NIPT in the second line are the sensitivity of current screening and the probability of having an invasive test after positive screening.

\section{DISCUSSION}

In Belgium, almost 100000 women participated in the current screening. Introducing NIPT as a contingent test or in first line is expected to reduce the number of procedure-related miscarriages. In addition, the number of T21 diagnoses missed by screening will be strongly reduced when NIPT is used in first line. Whereas NIPT as a contingent test at a price of $€ 460$ will lead to shortterm savings of about $€ 1.6$ million, NIPT in first line has a high impact on budgets, unless the price of NIPT is considerably reduced.

\section{Strengths and limitations of study}

The major strength of the model is the availability of context-specific real-world information and the ability to reflect the current Belgian screening situation by calibrating the model to the number of women screened, the expected and observed number of children born with Down syndrome and the number of invasive tests performed in Belgium. This calibration ensures that the initial screening model, including a large amount of real-world Belgian data on test characteristics, 
probabilities and costs, reflects the current Belgian screening situation as accurately as possible. This initial model is then used to construct the second and first line NIPT screening situation. The expected 219 births with Down syndrome if no screening is performed is used as a control variable and checked in all models and all simulations. Full details of the models are available in online supplementary material.

When NIPT is compared with the current screening system, NIPT is clearly superior in terms of sensitivity and specificity for the detection of T21 and other types of trisomy. Nevertheless, the model focuses on the detection of T21 and does not take into account the effects of screening for trisomy 13 (T13) and 18 (T18). Among the aneuploidy forms, T21 has the highest birth prevalence rate. ${ }^{19}$ T18 occurs less frequently and T13 is rather rare and survival of neonates with T13 or T18 beyond the first days of life is rare. ${ }^{20}$ The fetal fraction in T21 pregnancies is significantly higher compared with T13 and T18 pregnancies, which may help explain the higher sensitivity and specificity of NIPT for detecting T21. ${ }^{21}$ More research is needed to evaluate the use of primary NIPT to detect T13 and T18 which may lead to more invasive tests because of false-positive test results. If the current biochemical analyses are replaced by NIPT, the detection of some other chromosomal aberrations may be missed. ${ }^{22}$ At present, the clinical importance is unclear as a NT $>3.5 \mathrm{~mm}$ will already pick up many of these abnormalities. This is of relevance, as keeping in place the biochemical screening in parallel with NIPT would lead to a much less pronounced drop in invasive testing with a different impact on both costs and effects of the NIPT scenarios modelled.

The major weakness of the model is the inability to apply a long-term horizon and translate outcomes to incremental cost-effectiveness ratios expressing results in euros per (QA)LY gained. Two studies incorporate a lifetime cost of Down syndrome from a societal perspective of $\$ 940,000^{23}$ and $\$ 677,000,{ }^{24}$ respectively. A lifetime cost of Down syndrome of $\$ 900000$ is also mentioned by Cuckle $e t a l .{ }^{25}$ This amount is extrapolated from a 1992 average lifetime societal costs for an individual with Down syndrome of $\$ 451000 .^{26}$ The largest part $(64 \%)$ was due to indirect costs (productivity losses) which were calculated with the human capital approach. However, in contrast to the friction cost approach, this overestimates the total incremental cost for society. The friction-cost method, which is recommended by the Belgian guidelines for economic evaluations, ${ }^{3}$ is based on the idea that organisations need a certain time span (the friction period) to restore the initial production level after an employee becomes absent from work. The amount of production lost to society will be much lower than the above stated numbers and depends on the length of this friction period.

Furthermore, quality of life (QoL) is of major importance. One study included maternal QALYs in their analysis. ${ }^{23}$ The QoL data used in this study were based on studies of Kuppermann et $a t^{27-29}$ in women seeking genetic counselling and being less than 20 weeks pregnant. Their preferences, based on a hypothetical situation, might be very different from parents having a child with Down syndrome. Both the impact on life years (as a result of procedure-related or induced miscarriage) and QoL (eg, on parents during testing, people with/without Down syndrome and their parents) are not clear enough to make proper calculations with a long-term horizon. Furthermore, as stated by Petrou, ${ }^{30}$ "the matter is complicated further when one considers the positive utility effects that might accrue from a future 'replacement' child. The important point to note, however, is that an objective economic evaluation that measures and values the resource savings that follow the abortion of the affected fetus or unborn child requires a commensurate measurement and valuation of averted benefits. Furthermore, this remains the case whenever averted costs are incorporated into the evaluation, since the fetus or unborn child is necessarily ascribed a future human status that, by any measure, will have positive value and utility." There are also other relevant costs outside the healthcare system. "When the resource use implications for other sectors of society are considered the issue becomes more complicated: for example, the avoided excess costs associated with educational and institutional care, would need to be considered, as well as the costs of voluntary services and care incurred by the family." ${ }^{31}$ Gathering the necessary information on all these incremental elements could be the subject of future research.

In an ideal situation, all of these incremental elements would be taken into account. However, a translation into (QA)LYs gained was not performed because, within the time frame of this study, not enough reliable data could be gathered to work this out. This does not mean that we consider longer term costs and effects unimportant. On the contrary, we present the impact on various outcomes such as T21 detection, procedure-related pregnancy loss and total number of Down births whether or not after a false-negative screening test in a transparent way in order to inform our policymakers. Furthermore, if all harms (procedure-related pregnancy loss and Down birth after a false-negative screening result) are reduced and the cost per diagnosis stays the same, then it becomes difficult to oppose the introduction and reimbursement of this new technology.

\section{Comparison with other studies}

A systematic review of full economic evaluations on the cost-effectiveness of NIPT was performed in December 2013 by searching the websites of HTA institutes and the following databases: CRD HTA, CRD NHS EED, OVID MEDLINE and EMBASE. Details on the search strategy and selection process are available elsewhere. ${ }^{9}$ Seven full economic evaluations were retained. ${ }^{23-25} 32-35$ All studies were published recently (2011-2013). Five were performed in the USA, one in Australia ${ }^{33}$ and one in the 
UK. ${ }^{25}$ An additional economic evaluation from Ontario, Canada, was published during the writing of this article. $^{36}$

The comparator is different across the identified studies and results are as follows:

- Contingent screening with NIPT versus current practice. Contingent screening is more efficient than current standard of care, providing benefits at a lower cost. $^{24}{ }^{32}$ In one of these studies, cost savings were obtained by including a cost for Down syndrome. ${ }^{24}$ The only study without any explicit conflict of interest concludes that the introduction of NIPT for screening of high-risk pregnancies would result in better outcomes (additional T21 detected, reduced invasive testing and thus less procedure-related fetal losses), while costs would increase by about $10 \%$, which will need further policy planning. ${ }^{33}$

- Contingent screening with NIPT versus universal NIPT screening: Contingent screening is more efficient than universal screening. ${ }^{25} 35$ The cost for contingent screening is substantially lower than with universal screening. ${ }^{35}$ Offering NIPT to all women would only become affordable if the NIPT costs fall substantially. ${ }^{25}$

- Contingent screening with NIPT versus NIPT as a diagnostic tool: Contingent screening with NIPT is more efficient than applying NIPT as a diagnostic tool. ${ }^{23}$

Results of the previous studies are unfortunately not easily transferable to the Belgian context for several reasons. The populations described in the economic evaluations differ. Some model the general population of pregnant women ${ }^{25} 35$ while the other studies only include populations at high risk for T21. Related to this, the interventions and comparators used in the models differ. Not all studies consider NIPT in the first and second line. Only two studies include universal NIPT screening, ${ }^{25} 35$ of which one does not include the current situation. ${ }^{35}$ Furthermore, the values for several input variables are often not representative for the Belgian situation. For example, the sensitivity of first trimester combined screening $(85 \%)$ in the study of Song et $a l^{24}$ is much higher than in the real-world Belgian population. The focus of the economic evaluation lies in the first place on the number of T21 detected. However, when comparing the estimated number of children born with Down syndrome, one should be cautious about differences in, for example, pregnancy termination which is reported to be lower in, for example, the USA compared with Europe. ${ }^{37}$ As previously mentioned, inclusion of long-term costs and QoL data should also be supported by better data.

\section{The price of NIPT}

The price of NIPT varies widely across the economic evaluations published in 2012 or 2013: \$1200 (€880, $£ 713),{ }^{32} \$ 795$ (€583, £472), ${ }^{24}$ AU $\$ 743$ (€479, £388), ${ }^{33}$ and a price in the range of $\$ 500-\$ 2000$ ( $€ 367-€ 1466$, $£ 297-£ 1187) .{ }^{25}$ The costs to perform this test are decreasing. In Belgium, the official price of the University Hospital in Leuven is $€ 460$ ( $£ 373$ ). Sequenom has announced a low-cost NIPT of $\$ 250-\$ 300$ (€183-€220, £149-£178), to be available by the end of $2014 .^{38}$ These changes in prices, together with test accuracy, should be followed in order to take appropriate policy decisions.

\section{Pressure for referral to NIPT}

Most triage scenarios published as well as our model start from the combined ultrasound and biochemical screening. If reimbursement can be restricted to the 5\% of the screened population using the 1:300 cut-off, this may actually lead to a reduction in overall harms and savings for the healthcare budget, even at a cost per NIPT of $€ 460$. However, in this case, there will be pressure both from physicians and patients, to further lower the threshold for referral to NIPT, officially or informally. Indeed, in the absence of rigid quality assessment, the ultrasound part of the current screening remains strongly operator (and machine) dependent. This may lead to an increase in the number of women considered at risk after the current screening and thus eligible for NIPT reimbursement.

\section{Conditions for a successful introduction of NIPT}

Providing correct information and counselling and respect for the decision taken by the women or parents remains a cornerstone of any screening process.

As aforementioned the NIPT test does not provide a result in a fraction of women tested. If primary NIPT is offered at gestational week 10 the proportion of 'no result' after a repeat NIPT may be $4 \%$ instead of $2 \%$. If most of these women would opt directly for invasive testing instead of falling back to the current screening tests as we assumed, the reduction in harms related to the invasive procedure might not be realised. It is therefore crucial to monitor the performance of the real-life implementation of NIPT not only for sensitivity and specificity, but also for the proportion of 'no results' and the uptake of invasive testing after a 'no result' answer for NIPT in first line.

Several experts have expressed their fear that the quality of NT will decline once NIPT is broadly introduced. The ultrasound should remain a key component of the prenatal screening process also after the introduction of NIPT in second or first line. Women with a fetal NT $>3.5 \mathrm{~mm}$ (the 99th centile) are directly (without use of biochemistry information) offered genetic counselling, diagnostic invasive testing and follow-up in keeping with international guidelines. ${ }^{36}$ In such cases, there is a greater than $30 \%$ risk of chromosomal abnormalities, including but not limited to T21, ${ }^{17}$ and other abnormalities such as heart defects. ${ }^{39} 40$

It has repeatedly been recommended that NT-based risk assessment should only be implemented in centres with appropriately trained and accredited sonographers using high-quality equipment. Results should be subject 
to regular audit by an external agency. ${ }^{17} 40$ Such requirements are still to be implemented in Belgium. Also the calibration of the ultrasound machines seems to be a problem. ${ }^{41}$ For example, an NT of $3.5 \mathrm{~mm}$ is reported as $3.2 \mathrm{~mm}$ on one machine and as $3.8 \mathrm{~mm}$ on another instrument. This finding illustrates the clear need for further standardisation of the NT assessment. We believe that improving the quality of the ultrasound NT assessment in Belgium could increase the overall sensitivity of the screening, for example, from $72.5 \%$ to $77.5 \%$ at 95\% specificity. This improvement has been modelled separately and confirms that any improvement of the current screening sensitivity is mainly of importance when NIPT is used in the second line, reducing the number of T21 cases missed because of a false-negative result. It could also help in the acceptance of the current screening as an alternative test in cases where NIPT does not provide a result in first line screening. Amniocentesis and CVS carry a 1-2\% risk of membrane rupture, a $0.3 \%$ risk of sustained oligohydramnios, ${ }^{13}$ and a $1 \%$ risk of induced miscarriage, which may be higher after CVS as compared with amniocentesis. ${ }^{14}{ }^{42}$ It has been suggested that 100-400 CVSs are needed before the learning curve reaches a plateau. ${ }^{42}$ The risk may thus be lower in the hands of experienced operators and higher in low volume, less experienced centres. Currently, no required minimum volumes have been defined in Belgium and invasive testing is still performed in many small centres. Therefore, we applied a $1 \%$ risk of procedure-related miscarriage after CVS or amniocentesis.

\section{Conclusions and policy implications}

In comparison with the current prenatal screening for T21, the appropriate use of NIPT in either the first or second line clearly improves the benefit-risk ratio. Based on the availability of data, it was not possible to reliably calculate cost per (QA)LY gained. From an economic point of view, assuming that we accept the current screening situation, we recommend to our National Health Insurer to cover the cost of NIPT if the introduction of NIPT does not increase the screening cost per case of T21 detected. If offered at the current price of $€ 460$, NIPT can be introduced as a triage test, even if the screening risk cut-off is lowered from 1:300 to 1:600, corresponding to about $9 \%$ positive screen results eligible for NIPT reimbursement. Attention should be paid to further increase the quality of the current screening with NT. As the number of invasive diagnostic tests will most likely decrease, procedures should be centralised. In terms of benefits and harms, the use of NIPT in the first line is preferred over its use in the second line. However, the cost of NIPT should be lowered to about $€ 150$ in order not to increase the screening cost per case of T21 detected. In Belgium, at this (future) price level, NIPT should be offered to and reimbursed for all pregnant women.
Contributors MN, FH and WG have coauthored the health technology assessment report. All authors have been responsible for gathering the necessary data to perform this economic evaluation. MN and $\mathrm{FH}$ have independently performed the modelling exercise. All authors have participated in writing the document, revising the draft paper and approved the version to be published. MN is the guarantor.

Funding The project was funded by KCE as part of its annual programme. Competing interests None.

Provenance and peer review Not commissioned; externally peer reviewed.

Data sharing statement No additional data are available.

Open Access This is an Open Access article distributed in accordance with the Creative Commons Attribution Non Commercial (CC BY-NC 4.0) license, which permits others to distribute, remix, adapt, build upon this work noncommercially, and license their derivative works on different terms, provided the original work is properly cited and the use is non-commercial. See: http:// creativecommons.org/licenses/by-nc/4.0/

\section{REFERENCES}

1. Benn P, Borell A, Chiu R, et al. Position statement from the Aneuploidy Screening Committee on behalf of the Board of the International Society for Prenatal Diagnosis. Prenat Diagn 2013;33:622-9.

2. Bianchi DW, Parker RL, Wentworth J, et al. DNA sequencing versus standard prenatal aneuploidy screening. $N$ Engl J Med 2014;370:799-808.

3. Cleemput I, Neyt M, Van de Sande S, et al. Belgian guidelines for economic evaluations and budget impact analyses: second edition. Health Technology Assessment (HTA). Brussels: Belgian Health Care Knowledge Centre(KCE), 2012.

4. Mutton D, Alberman E, Hook EB. Cytogenetic and epidemiological findings in Down syndrome, England and Wales 1989 to 1993. National Down Syndrome Cytogenetic Register and the Association of Clinical Cytogeneticists. J Med Genet 1996;33:387-94.

5. Boyle B, Morris J, McConkey R, et al. Prevalence and risk of Down syndrome in monozygotic and dizygotic multiple pregnancies in Europe: implications for prenatal screening. BJOG 2014;121:809-19.

6. Snijders RJ, Sundberg K, Holzgreve W, et al. Maternal age- and gestation-specific risk for trisomy 21. Ultrasound Obstet Gynecol 1999;13:167-70.

7. Avalos A, Galindo C, Li DK. A systematic review to calculate background miscarriage rates using life table analysis. Birth Defects Res A Clin Mol Teratol 2012;94:417-23.

8. Morris JK, Alberman E, Mutton D, et al. Cytogenetic and epidemiological findings in Down syndrome: England and Wales 1989-2009. Am J Med Genet A 2012;158A:1151-7.

9. Hulstaert F, Neyt M, Gyselaers W. The non-invasive prenatal test (NIPT) for trisomy 21-health economic aspects. Health Technology Assessment (HTA). Brussels: Belgian Health Care Knowledge Centre(KCE), 2014

10. Benn P, Cuckle H, Pergament E. Non-invasive prenatal testing for aneuploidy: current status and future prospects. Ultrasound Obstet Gynecol 2013;42:15-33.

11. Saucedo MC, DeVigan C, Vodovar V, et al. Measurement of nuchal translucency and the prenatal diagnosis of Down syndrome. Obstet Gynecol 2009;114:829-38.

12. Harris RA, Washington AE, Nease RF Jr, et al. Cost utility of prenatal diagnosis and the risk-based threshold. Lancet 2004;363:276-82.

13. Richter J, Henry A, Ryan G, et al. Amniopatch procedure after previable iatrogenic rupture of the membranes: a two-center review. Prenat Diagn 2013;33:391-6.

14. Tabor A, Philip J, Madsen $\mathrm{M}$, et al. Randomised controlled trial of genetic amniocentesis in 4606 low-risk women. Lancet 1986;1:1287-93.

15. Alfirevic Z, Sundberg K, Brigham S. Amniocentesis and chorionic villus sampling for prenatal diagnosis. Cochrane Database Syst Rev 2003(3):CD003252.

16. Choi H, Van Riper M, Thoyre S. Decision making following a prenatal diagnosis of Down syndrome: an integrative review. J Midwifery Womens Health 2012;57:156-64.

17. Snijders RJ, Noble P, Sebire N, et al. UK multicentre project on assessment of risk of trisomy 21 by maternal age and fetal nuchal-translucency thickness at 10-14 weeks of gestation. Fetal Medicine Foundation First Trimester Screening Group. Lancet 1998;352:343-6. 
18. Briggs A, Sculpher M, Claxton K. Decision modelling for health economic evaluation. Oxford: Oxford University Press, 2006.

19. Wellesley D, Dolk H, Boyd PA, et al. Rare chromosome abnormalities, prevalence and prenatal diagnosis rates from population-based congenital anomaly registers in Europe. Eur $J$ Hum Genet 2012;20:521-6.

20. Houlihan OA, O'Donoghue K. The natural history of pregnancies with a diagnosis of Trisomy 18 or Trisomy 13; a retrospective case series. BMC Pregnancy Childbirth 2013;13:209.

21. Rava RP, Srinivasan A, Sehnert AJ, et al. Circulating fetal cell-free DNA fractions differ in autosomal aneuploidies and monosomy $X$. Clin Chem 2013;60:243-50.

22. Petersen $\mathrm{O}$, Vogel I, Ekelund $\mathrm{C}$, et al. Potential diagnostic consequences of applying non-invasive prenatal testing (NIPT); a population-based study from a country with existing first trimester screening. Ultrasound Obstet Gynecol 2013;43:265-71.

23. Ohno M, Caughey A. The role of noninvasive prenatal testing as a diagnostic versus a screening tool-a cost-effectiveness analysis. Prenatal Diagnosis 2013;33:630-5.

24. Song K, Musci TJ, Caughey AB. Clinical utility and cost of non-invasive prenatal testing with cfDNA analysis in high-risk women based on a US population. J Matern Fetal Neonatal Med 2013;26:1180-5.

25. Cuckle H, Benn P, Pergament E. Maternal cfDNA screening for Down syndrome: a cost sensitivity analysis. Prenat Diagn 2013:33:636-42.

26. Waitzman N, Roman P, Scheffler R, et al. Economic costs of birth defects and cerebral palsy-United States, 1992. MMWR Morb Mortal Wkly Rep 1995;44:694-9.

27. Kuppermann M, Nease RF, Learman LA, et al. Procedure-related miscarriages and Down syndrome-affected births: implications for prenatal testing based on women's preferences. Obstet Gynecol 2000;96:511-16.

28. Kuppermann M, Nease RF Jr, Gates $\mathrm{E}$, et al. How do women of diverse backgrounds value prenatal testing outcomes? Prenat Diagn 2004;24:424-9

29. Kuppermann M, Feeny D, Gates E, et al. Preferences of women facing a prenatal diagnostic choice: long-term outcomes matter most. Prenat Diagn 1999;19:711-16.
30. Petrou S. Methodological limitations of economic evaluations of antenatal screening. Health Econ 2001;10:775-8.

31. Brown J, Buxton M. The economic perspective. Br Med Bull 1998;54:993-1009.

32. Garfield SS, Armstrong SO. Clinical and cost consequences of incorporating a novel non-invasive prenatal test into the diagnostic pathway for fetal trisomies. J Manag Care Med 2012;15:32-9.

33. O'Leary P, Maxwell S, Murch A, et al. Prenatal screening for Down syndrome in Australia: costs and benefits of current and novel screening strategies. Aust N Z J Obstet Gynaecol 2013;53:425-33.

34. Palomaki GE, Kloza EM, Lambert-Messerlian GM, et al. DNA sequencing of maternal plasma to detect Down syndrome: an international clinical validation study. Genet Med 2011; 13:913-20.

35. Wald NJ, Bestwick JP. Incorporating DNA sequencing into current prenatal screening practice for Down's syndrome. PLOS ONE 2013;8:e58732.

36. Okun N, Teitelbaum M, Huang T, et al. The price of performance: a cost and performance analysis of the implementation of cell-free fetal DNA testing for Down syndrome in Ontario, Canada. Prenat Diagn 2014;34:350-6.

37. Natoli JL, Ackerman DL, McDermott S, et al. Prenatal diagnosis of Down syndrome: a systematic review of termination rates (19952011). Prenat Diagn 2012;32:142-53.

38. GenomeWeb staff reporter. Sequenom Officials Discuss Plans for Low-Cost NIPT, 17 January 2014.

39. Nicolaides KH. Nuchal translucency and other first-trimester sonographic markers of chromosomal abnormalities. Am J Obstet Gynecol 2004;191:45-67.

40. Chitayat $\mathrm{D}$, Langlois $\mathrm{S}$, Wilson RD. Prenatal screening for fetal aneuploidy in singleton pregnancies. J Obstet Gynaecol Can 2011:33:736-50.

41. Axell RG, Gillett A, Pasupathy D, et al. The accuracy of nuchal translucency measurement depends on the equipment used and its calibration. Ultrasound Obstet Gynecol 2014:44:31-7.

42. Tabor A, Alfirevic $Z$. Update on procedure-related risks for prenatal diagnosis techniques. Fetal Diagn Ther 2010;27:1-7. 